\title{
Dual Ultrastructural Localization of Two Neurotransmitter-related Antigens: Colloidal Gold-labeled Neurophysin-immunoreactive Supraoptic Neurons Receive Peroxidase-labeled Glutamate Decarboxylase- or Gold-labeled GABA-immunoreactive Synapses ${ }^{1}$
}

\author{
ANTHONY N. VAN DEN POL
}

Section of Neurosurgery, Yale University Medical School, New Haven, Connecticut 06510

\begin{abstract}
To study the morphological substrate for interaction between two chemically distinct neuronal types, two double ultrastructural immunolabeling strategies were employed. In the first, two different electron-dense markers were used to examine simultaneously two different neurotransmitter-related antigens in the hypothalamic supraoptic nucleus in the same thin section. Results obtained with the first method were confirmed with a second approach based on postembedding immunostaining of alternate serial thin sections with different antisera. Antiserum against glutamate decarboxylase, the enzyme responsible for the synthesis of the inhibitory amino acid transmitter $\gamma$-aminobutyric acid (GABA), or antisera against GABA, was used to localize immunoreactive axons in the hypothalamic supraoptic nucleus. With light microscopy, glutamate decarboxylase- and GABA-immunoreactive axon terminals immunostained with peroxidase were found arborizing throughout all areas of the nucleus; terminal boutons were found adjacent to unlabeled somata within the nucleus. Cells containing immunoreactive oxytocin, vasopressin, and neurophysin were localized with peroxidase.

Glutamate decarboxylase-immunoreactive axons stained with peroxidase prior to embedding in plastic were demonstrated to contact neurons which contained vesicles immunostained with neurophysin antiserum by a post-embedding immunocytochemical procedure which used immunoglobulins or protein A adsorbed to colloidal gold as a second ultrastructural marker. Quantitative evaluation of postembedding staining with colloidal gold using a neurophysin primary antiserum indicated a specific antigen localization in neurosecretory vesicles. A critical factor in this double-labeling paradigm was that immunological reagents used in the second series did not cross-react with those used in the first series, regardless of the species of origin of antisera. To provide further verification of GABAergic synapses on neurophysin-containing neurons, alternate serial ultrathin sections were stained with colloidal gold using antisera
\end{abstract}

Received December 7, 1984; Revised May 13, 1985;

Accepted May 13, 1985

${ }^{1}$ I thank Dr. M. Sofroniew for neurophysin antiserum, Drs. W. Oertel, D. Schmechel, I. Kopin, and M. Tappaz for glutamate decarboxylase antiserum, Dr. P. Somogyi for GABA antiserum, and G. Collins and K. Szigeti for histological assistance. Support was provided by National Institutes of Health Grants NS 16296 and 10174 against either neurophysin or GABA; boutons immunoreactive for GABA made synaptic contact with supraoptic neurons containing neurophysin immunoreactivity. Converging results obtained with these two procedures indicate that GABAergic axons synapse directly on neurons containing oxytocin or vasopressin in the rat hypothalamic supraoptic nucleus. As boutons immunoreactive for glutamate decarboxylase are often found in synaptic contact with neurosecretory cells in the supraoptic nucleus, the inhibitory amino acid GABA may play a major role in the control of nonapeptides released by the supraoptic nucleus in the posterior pituitary.

To test the versatility of post-embedding immunostaining with colloidal gold, a number of other hypothalamic neuroactive substances, including prolactin, luteinizing hormone-releasing hormone, ACTH, $\alpha$-melanocyte-stimulating hormone, $\beta$-endorphin, and somatostatin, were localized in material treated with a number of different fixatives including glutaraldehyde and osmium. The approaches to double immunostaining described here should work with a large number of pairs of antigens in which the first can be stained with preembedding peroxidase and the second with post-embedding gold particles, or in which both can be stained with postembedding immunocytochemistry.

Immunocytochemical analysis of putative neurotransmitters in the central nervous system has provided insight into transmitter-specific localizations and projections. A further extension of immunocytochemical analysis of the brain using double immunolabeling for two different neurotransmitters allows the elucidation not only of general pathways, but also of the specific neurochemical connections made between two different types of neurons. Simultaneous immunostaining of two different antigens requires the use of two easily discriminable and electron-dense markers. Furthermore, the first series of immunoreagents should not cross-react with the second series. One dual ultrastructural immunocytochemical methodology using peroxidase as a pre-embedding marker for one transmitter and colloidal gold as a post-embedding marker for a second neurotransmitter is described here, and new information about synaptic relationships in the hypothalamic supraoptic nucleus (SON) is presented.

Immunocytochemistry with peroxidase as a light- and electrondense label has been the most prevalent approach to antigen localization in the brain, particularly for ultrastructural analysis. Colloidal gold as an immunolabel has been used in the peripheral nervous system (Probert et al., 1981; Larsson and StengaardPedersen, 1982) and in other tissue (Roth et al., 1978; Horisberger, 1979; DeMey et al., 1981; van den Pol, 1984a), but surprisingly little 
has been done with this useful marker in the central nervous system. Colloidal gold immunolabels are relatively easy to prepare, and they have a punctate high electron density which, unlike peroxidase, does not obscure general ultrastructural detail. Recent advances in silver intensification of gold may provide a degree of sensitivity comparable to or exceeding that of peroxidase methods (Holgate et al., 1983; van den Pol, 1985a, b). Colloidal gold can be quantitatively evaluated much more readily than enzymatic (e.g., peroxidase) markers. These factors, together with the use of colloidal gold as a second marker for double ultrastructural immunocytochemistry, make colloidal gold a valuable immunolabel which merits greater use in the central nervous system.

Neurons of the hypothalamic SON synthesize oxytocin, vasopressin, and their related neurophysins (NPHs). The primary axonal projection from these cells terminates in the posterior pituitary. Oxytocin and vasopressin immunoreactivity is also found in boutons terminating in many areas of the brain; these terminals originate primarily from the paraventricular (vasopressin or oxytocin) or suprachiasmatic nucleus (vasopressin) (Buijs, 1978; Nilaver et al., 1980; Swanson and Sawchenko, 1983 (review); van den Pol and Tsujimoto, 1985). In the SON, in addition to magnocellular neurons containing vasopressin and oxytocin, several reports have suggested the presence of both parvocellular neurons (LuQui and Fox, 1976; Felton and Cashner, 1979) and neurons which contain immunoreactivity for other substances, including tyrosine hydroxylase (van den Pol et al., 1984) and prolactin (Toubeau et al., 1979). Previous electrophysiological work (Nicoll and Barker, 1971; Moss et al., 1972; Bioulac et al., 1978) has indicated that neurons of the SON may have GABA receptors and that they respond to application of exogenous GABA. To determine whether GABAergic axons terminate directly on neuropeptide-secreting cells of the SON, two different approaches were used: the first allowed simultaneous examination of peroxidaselabeled boutons in contact with gold-labeled neurons in the same thin section, and the second utilized serial thin sections immunostained with different antisera to demonstrate synaptic contact between two chemically different neuron types.

\section{Materials and Methods}

Pre-embedding immunostaining with peroxidase. An antibody against the GABA-synthesizing enzyme glutamate decarboxylase (GAD) was used to localize axonal terminals containing the amino acid neurotransmitter GABA. After perfusion fixation of $0.9 \%$ saline, followed by a combination of $4 \%$ paraformaldehyde and $0.1 \%$ glutaraldehyde in a $0.1 \mathrm{~m}$ phosphate buffer, the hypothalami of adult male rats were immersed in $10 \%$ followed by $20 \%$ sucrose until the blocks sank. Blocks were then frozen in liquid nitrogen, thawed, and frozen once more to break membranes and thereby facilitate penetration of immunoglobulins into thick sections. Triton $X-100$, also used to damage membranes, appeared to give a greater loss of ultrastructural integrity than did freezing and was not generally used in these experiments. Hypothalamic blocks containing the SON were cut in $30-\mu \mathrm{m}$ coronal or horizontal sections with a Lancer Vibratome. After incubation in a blocking serum containing $5 \%$ normal rabbit serum, $1 \%$ bovine serum albumin (BSA), $0.1 \%$ glycine, and $0.1 \%$ lysine, sheep anti-rat GAD (gift of Drs. W. Oertel, D. Schmechel, M. Tappaz, and I. Kopin) was diluted to 1:1500 and was localized with a biotin-conjugated, affinity-purified rabbit anti-sheep antiserum followed by a molecular complex of avidin, biotin, and horseradish peroxidase (Vector Laboratories) (Hsu et al., 1981). After staining for GAD, sections were osmicated in $1 \%$ osmium tetroxide, dehydrated through a graded series of ethanol and propylene oxide, and flat embedded in Epon on glass slides with glass coverslips pretreated with Liquid Release Agent (Electron Microscopy Sciences), which facilitated removal of the glass for subsequent reembedding in Epon in a Beem capsule. Peroxidase-stained cells were best studied under glass but could also be readily examined after final embedding in a Beem capsule.

Additional sections were immunostained with an antiserum against GABA (gift of Dr. P. Somogyi) to confirm the presence of this amino acid in axons in the SON.

Sections were also stained using the pre-embedding peroxidase procedure with antisera against oxytocin (Immunonuclear Corp.), vasopressin (Immunonuclear), luteinizing hormone-releasing hormone (LHRH) (gift of $\mathrm{Dr}$.
R. Benoit), and NPH (gift of Dr. M. Sofroniew). Specificity controls for these antisera are described elsewhere (van den Pol and Tsujimoto, 1985).

Post-embedding immunostaining with colloidal gold:neurophysin. Two major problems that can be anticipated in ultrastructural immunocytochemistry are the possible inactivation of the antigen by the fixative, and the ability of the antigen to survive plastic embedding, yet still be accessible for immunoglobulin binding. During the course of these experiments, a number of fixatives and plastics were used to find the optimal conditions for immunochytochemical labeling which would also allow a reasonable retention of normal and visible ultrastructure. As high concentrations of glutaraldehyde $(>0.4 \%)$ eliminated pre-embedding immunostaining with antisera against neurophysin, and high concentrations of paraformaldehyde (4\%) were compatible with positive staining, the amount of glutaraldehyde added to $4 \%$ paraformaldehyde was varied from $0.05 \%$ to $1 \%$. Some tissue was not osmicated, whereas other tissue from the same brains was immersed in $1 \%$ osmium tetroxide in $0.1 \mathrm{~m}$ phosphate buffer for $40 \mathrm{~min}$.

To determine what embedding conditions allowed reasonable post embedding immunostaining and an ultrastructural appearance suitable for low magnification scanning, a number of plastics were used including Epon, Durcupan, LR White, and Lowicryl K4M. Embedding procedures for Epon and Durcupan were similar. Tissue embedded in LR White was dehydrated in ethanol before immersion in two 10-min changes of LR White; sections were embedded in closed capsules at $60^{\circ} \mathrm{C}$. Tissue embedded in Lowicryl was embedded according to the rapid procedure described by Altman et al. (1984). The total time after removal of the brain for dehydration, embedding, and hardening was only $3 \mathrm{hr}$. Thin sections could be examined in the electron microscope $4 \mathrm{hr}$ after fixative perfusion of the brain. Tissue was dehydrated in ethanol or dimethyl formamide, immersed in Lowicryl, and cured under ultraviolet radiation for $1 \mathrm{hr}$ in a cold room kept at $4^{\circ} \mathrm{C}$.

Pale gold or silver thin sections were cut on a Reichert OM U3 ultramicrotome and picked up on nicket or gold mesh grids or formvar-coated slot grids. All immunostaining was done in Falcon 60-well tissue culture plates (no. 3034 Microtest tissue culture plates). Each well has an identity number and holds about 20 to $25 \mu$ l of solution; a lid prevents excessive evaporation and keeps the yrids dust-free. These tissue culture plates are discarded after a single use. Grids were totally immersed in water for $5 \mathrm{~min}$ and then in a blocking buffer consisting of Tris-buffered saline (TBS) at a $\mathrm{pH}$ of 8.2 with $0.1 \%$ bovine serum albumin. TBS buffer was passed through a $0.22-\mu \mathrm{m}$ pore-size filter prior to use. If colloidal gold adsorbed to a goat anti-rabbit lgG was to be used, the blocking serum included $10 \%$ normal goat serum; in contrast, if protein A adsorbed to gold was used, the blocking buffer contained $1 \% \mathrm{BSA}$, and $0.1 \%$ glycine and $0.1 \%$ lysine. Grids were immersed in $20 \mu \mathrm{l}$ of NPH antiserum at a dilution of 1:400 to 1:1800 in TBS buffer for 1 to $16 \mathrm{hr}$. Grids were then placed in an LKB grid holder box with holes drilled through the top and bottom. The holes were slightly smaller than the diameter

TABLE I

Colloidal gold and peroxidase pre- and post-embedding double-labeling protocol

1. FIKATION (4T PARAF ORMALDEHYOE, $0.1 \%$ GLUTARALDEHYDE)

2 SUCROSE CRYOPROTECTION (20\% SUCROSE) / NITROGEN FREEZE THAW

3. CUT $30 \mu \mathrm{m}$ SECTIONS ON VIBRATOME

4. BLOCKING BUFFER (NORMAL RABBIT SERUM) ( 1 HOUR)

5. PRIMARY ANTISERUM (SHEEP ANTI-GLUTAMATE DECARBOXYLASE) (OVERNIGHT)

6 WASH

7. BIOTINYLATED SECONDARY (BIOTIN-RABEIT ANTI-SHEEP IGG) (3O MIN)

8. WASH

9. AVIDIN-BIOTIN PEROXIDASE COMPLEX (3O MIN)

10. WASH

11 OSMICATE ( $1 \%$ OSMIUM TETROXIDE/ 40 MIN)

12. DEHYORATE, EMBEDDED IN EPON ON GLASS

13. EXAMINE THICK SECTIONS WITH LIGHT MICROSCOPE /PHOTOGRAPH OR DRAW

4 REMOVE GLASS

15. CUT OUT AREA OF INTEREST WITH RAZOR BLADE

16. RE-EMBED IN EPON IN BEEM CAPSULE

17. SELECT AREA TO BE THIN SECTIONED, AND TRIM ACCORDINGLY

18. SAVE THIN SECTIONS ON GOLD OR NICKEL GRIOS

19. PUT GRIDS IN WATER IN FALCON TISSUE CULTURE PLATE

20 BLOCKING BUFHER IBS (NURMAL GUAT SERUM)

21. SECOND PRIMARY ANTISERA (ANT I-NEUROPHYSIN)

22. WASH IN TES IN LKB GRID HOLDER IN BEAKER WITH STIRRING

23. GDAT ANT I-RADDIT IGG (OR PROTEIN A) ADSORBED TO GOLO

24. WASH IN TBS IN LKB GRIO HOL DER

25 WASH IN WATER

26. DRV

27. STAIN LIGHTLY (LEAD CITRATE AND URANYL ACETATE)
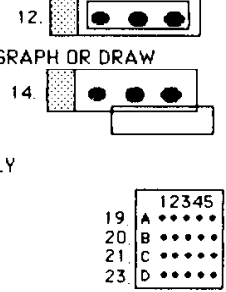

$23 . \mid \mathrm{C}, \ldots .$.

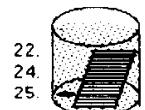


of the grid. The entire grid box was placed in a beaker of buffer for washing in between antibody or protein A steps; continuous buffer movement was produced by a small stirring bar placed at the bottom of the beaker. Grids were washed for $1 \mathrm{hr}$. Grids were then incubated in either affinity-purified goat anti-rabbit immunoglobulin (heavy and light chain) or protein $A$ adsorbed to 5-, 10-, or 20-nm colloidal gold particles for $2 \mathrm{hr}$ and then washed in buffer followed by water. A brief post-fixation in $0.2 \%$ glutaraldehyde may facilitate adherence of the protein-bound colloidal gold particles to the thin section.
Figure 1. Pre-embedding immunostaining in the supraoptic nucleus. $A, \mathrm{NPH}$ antiserum stains both neurons and large processes of the SON. $B, G A D$ antiserum stains axons throughout the SON. $C$, As a negative control, LHRH antiserum was used. Axons and cells rostral to the SON were stained, but little staining is found within the SON as seen here. $D$, GABA antiserum stains axons surrounding large neurons in the SON, as seen on this semithin section. Bars, $30 \mu \mathrm{m}$.
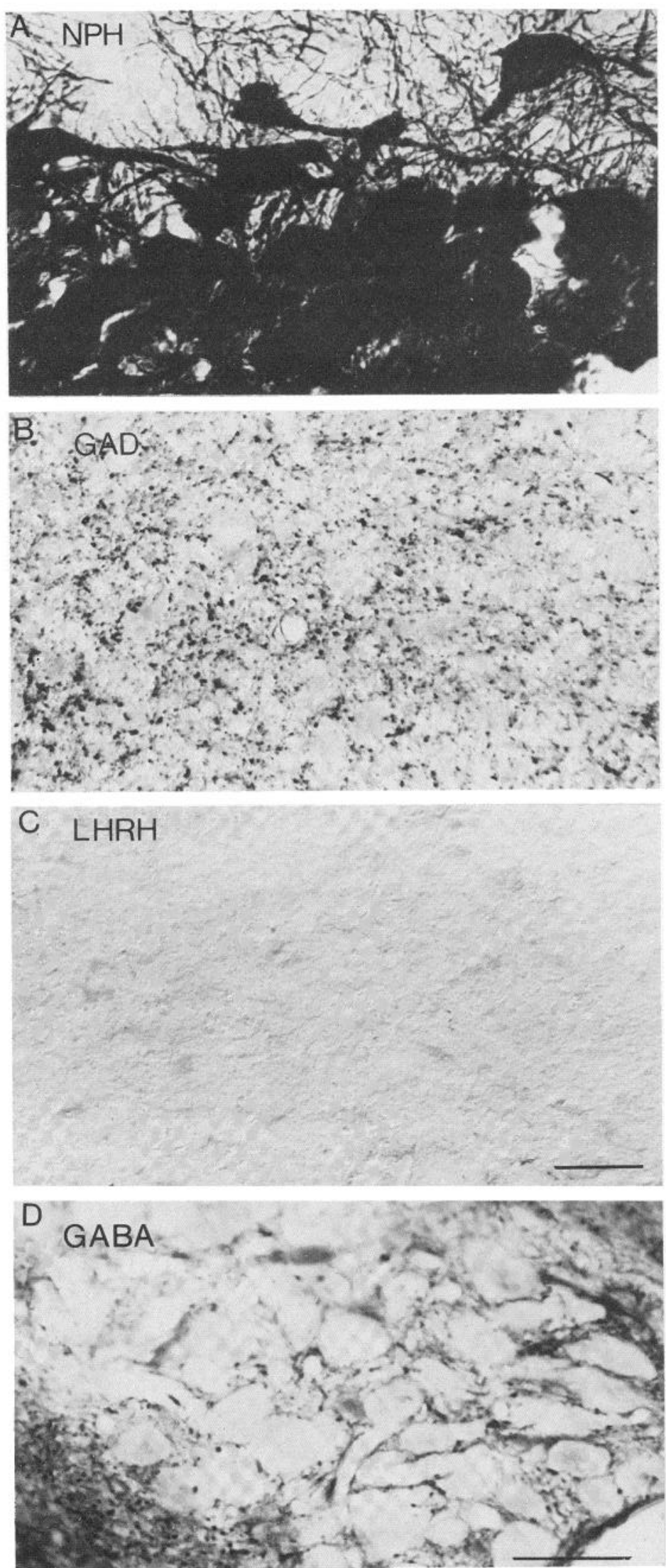

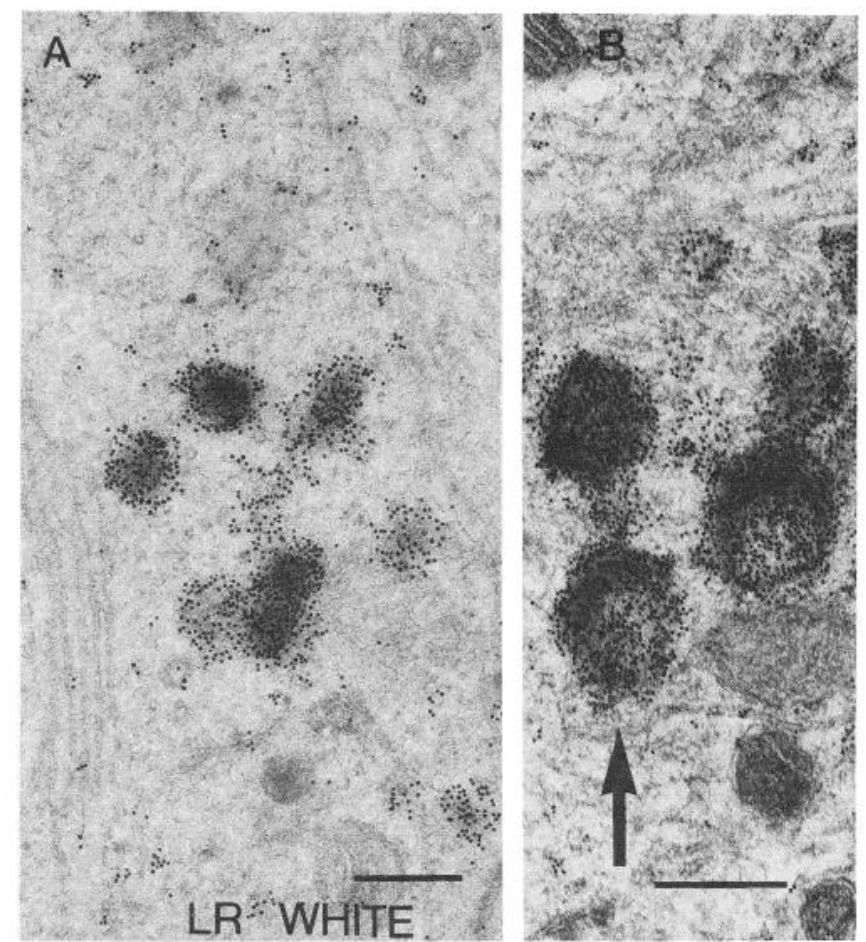

After immunostaining, sections on grids were counterstained with uranyl acetate, tannic acid, lead citrate, or some combination of the three. Tissue was examined in a Philips EM 201 with accelerating voltages of $40 \mathrm{kV}$ for lightly counterstained sections, or $60 \mathrm{kV}$ for more contrasted specimens.

Post-embedding immunostaining with colloidal gold:alternate ultrathin sections stained with GABA or NPH antisera. From brains perfused with $4 \%$ paraformaldehyde and $1 \%$ glutaraldehyde and later immersed in $1 \%$ osmium tetroxide, serial thin Epon sections were cut. Groups of three sections were picked up on either formvar-coated single-slot grids or 300 mesh nickel grids. Alternate grids were stained with GABA antiserum (1:6000) or NPH antiserum (1:1200). Because post-embedding immunostaining may result in partial reduction in the intensity of staining of both membranes and synaptic membrane specializations, additional grids in a series of thin sections were not used for immunostaining but were stained instead with uranyl acetate and lead citrate. Mesh grids allowed immunostaining of both the top and bottom surfaces of a thin section, but grid bars obstructed the view of certain regions of tissue. Formvar-coated slot grids allowed a view of the entire thin section, but immunostaining could only be done on the top surface of the section, as formvar on the bottom surface eliminated immunostaining there. Thus, mesh grids commonly had approximately twice the colloidal gold label over immunoreactive structures that slot grids had.

Preparation of colloidal gold-adsorbed proteins. Different procedures were used to form the different size colloidal gold particles. Particularly important during preparation of the gold sol is that all glassware must be extremely clean. Larger gold particles can be prepared with reduction of gold salt with sodium citrate (Frens, 1973) or ascorbate (Horisberger and Vonlanthen, 1978), while the smallest gold particles ( $5 \mathrm{~nm}$ ) can be prepared with white phosphorus and ether (Romano et al., 1974); care should be taken during preparation of the colloidal gold particles with the white phosphorus-ether method as the mixture is explosive. Detailed procedures of colloidal gold preparation can be found elsewhere (see references in van den Pol, 1984a; 1985b).

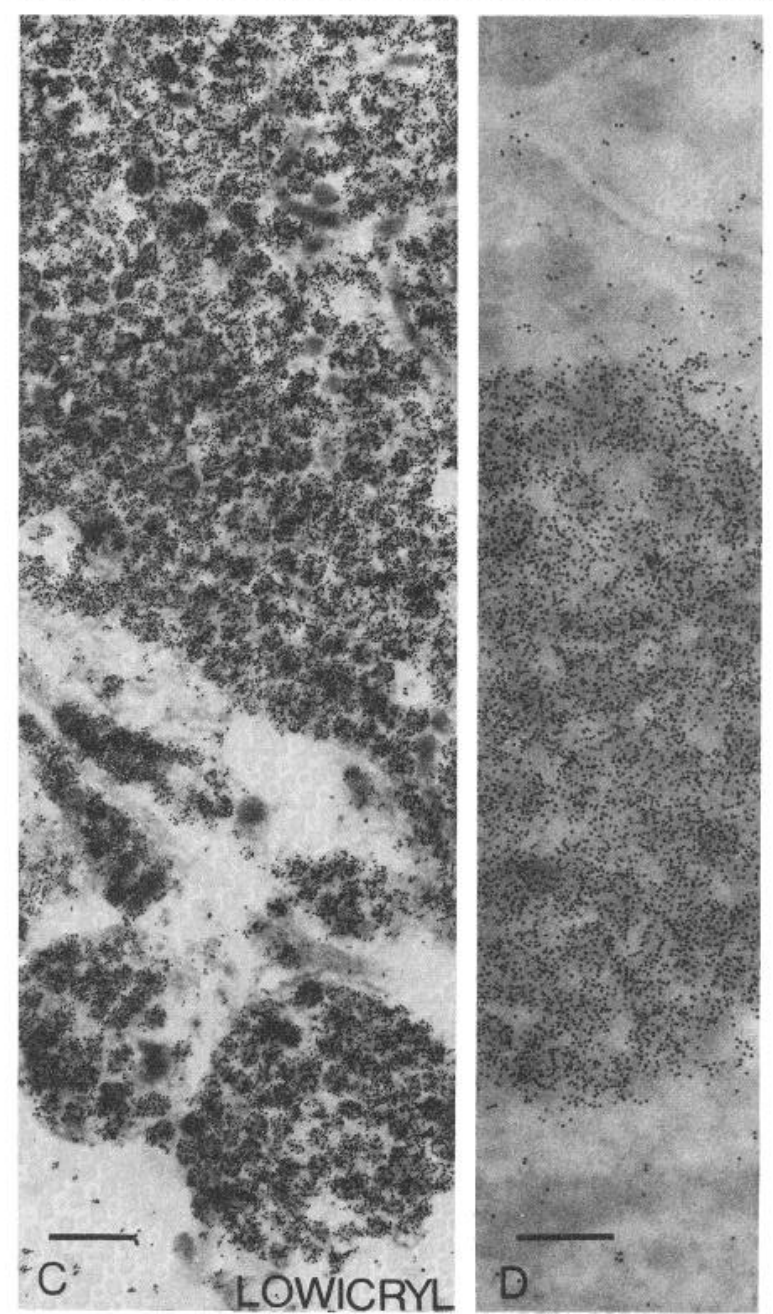

Adsorption of immunoglobulins and protein A to colloidal gold. Colloidal gold particles have a negative charge and adsorb readily to many proteins, including immunoglobulins and protein A. Protein A (Pharmacia), derived from Staphylococcus aureus, has a high affinity for the Fc part of some species of $\lg G$ and therefore makes a useful ligand for immunocytochemical localization of antigens. Critical factors in adsorption of gold particles to proteins are the size of the gold particle and the size, isoelectric point, and nature of the protein to be adsorbed, as discussed elsewhere (Roth et al., 1978; Horisberger, 1979; DeMey et al., 1981; van den Pol, 1984a).

Immunocytochemistry controls. Additional characterization and discussion of antisera specificity are reviewed elsewhere for the NPH (Sofroniew and Weindl, 1978), GAD (Oertel et al., 1981a, b; Tappaz et al., 1982, 1983), and GABA (Hodgson et al., 1985; Somogyi et al., 1985) antisera.

Antigen adsorbtion controls with NPH (Sofroniew and Weindl, 1978; van den Pol, 1982), GAD (Oertel et al., 1981a, b), and GABA (Hodgson et al., 1985; Somogyi and Hodgson, 1985) have been previously discussed. As further controls, preimmune serum, or normal serum, or antisera against proteins or peptides known to exist in other neural loci were substituted for the specific primary antiserum.

For the post-embedding colloidal gold immunostaining, the localization of immunoreactivity in the SON and neurohypophysis was compared in different plastics. Immunoreactivity of large neurosecretory vesicles of axons in the neurohypophysis were compared with staining of secretory granules in the intermediate and anterior lobe of the pituitary. GAD antiserum was substituted for NPH antiserum, with the necessary modification of secondary antisera. Post-embedding staining with GABA antiserum was done in the presence of $\mathrm{BSA}$, the carrier to which the GABA hapten was bound to produce the antigen, and after absorption with a GABA-BSA conjugate (Table I).

\section{Results}

Light microscopy: Pre-embedding immunostaining. Within the SON, immunoreactive perikarya, dendrites, and axons were found with pre-embedding peroxidase immunostaining with antisera against oxytocin, vasopressin, and NPH (Fig. 1A). GAD- and GABAimmunoreactive axons labeled with peroxidase were found throughout the SON with light microscopy (Fig. 1, B and D). As a control, immunostaining with an antiserum against LHRH was used; although

embedded in LR White. In both low $(C)$ and higher $(D)$ magnifications, intense gold staining of vesicles in the neurohypophysis can be seen in Lowicryl-embedded tissue. Protein appears with a slight positive stain, while membranes exhibit negative staining. Bars: $A, 275 \mathrm{~nm} ; B, 250 \mathrm{~nm}, C, 450$ $\mathrm{nm} ; 0,300 \mathrm{~nm}$.

Figure 2. Post-embedding immunostaining with LR White and Lowicry K4M. A, Gold-labeled vesicles in process in the SON. B, Vesicle (arrow) immunostained with about 250 five-nanometer gold particles. $A$, and $B$ are 
Figure 3. Osmicated tissue embedded in Epon and treated with $10 \%$ hydrogen peroxide for $10 \mathrm{~min}$. A, Even at low electron microscopic magnifications, immunostained vesicles are clearly visible in a cell body. Immunoreactive vesicles can be identified with $\times 1500$ magnification. Both 10- $(B)$ and 5-nm $(C)$ particles stain the vesicles in the magnocellular neuron dark, sometimes so densely that the particles form a solid dark field over the middle of the vesicles. Although the immunostaining is optimal, the ultrastructure has been seriously compromised by the hydrogen peroxide treatment. NU, nucleus; $M$, mitochondrion. Bars: $A, 400$ $\mathrm{nm} ; B, 180 \mathrm{~nm} ; C, 180 \mathrm{~nm}$.
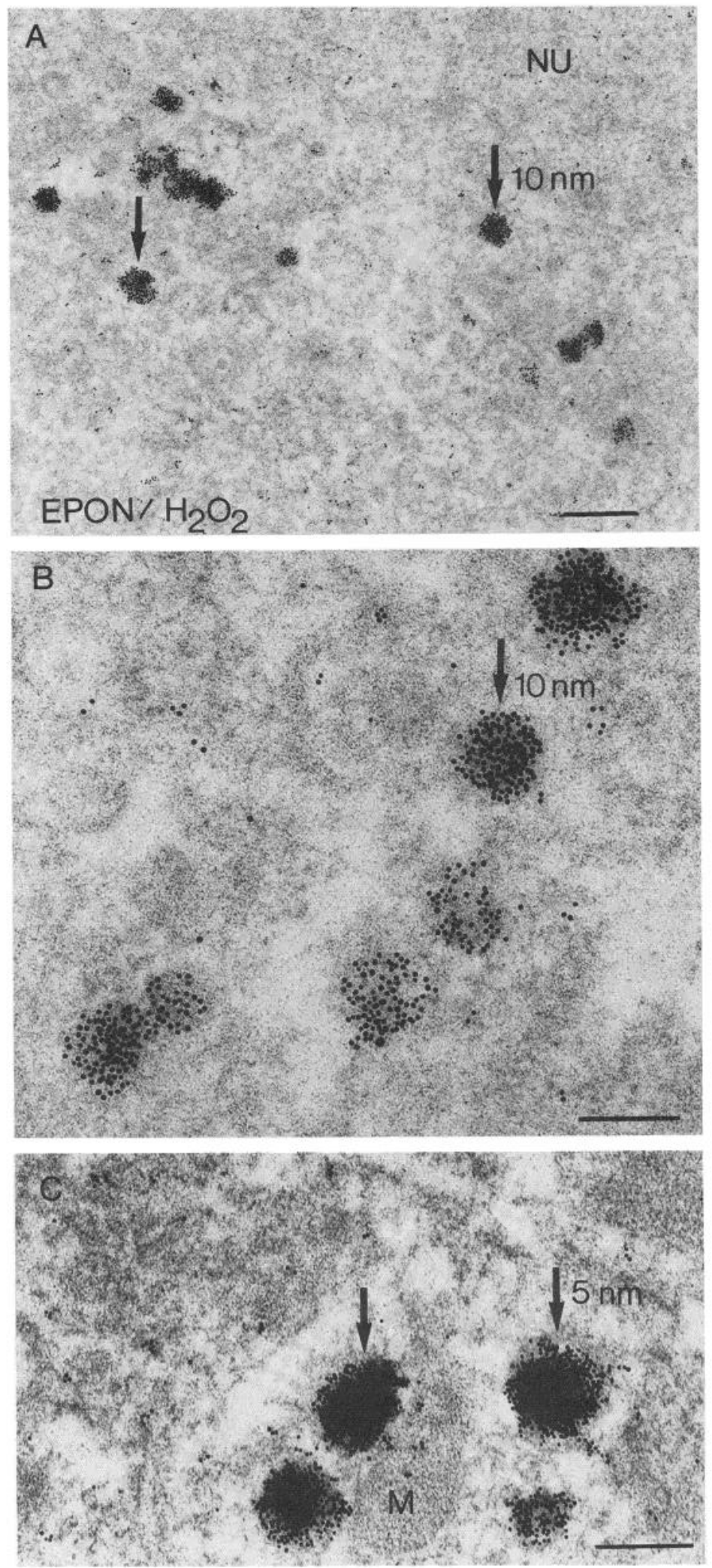

Figure 4. SON and neurohypophysis embedded in Epon after osmium treatment. Large, 20-nm gold particles are easier to see in $B$, but the number of small, 5-nm particles over single vesicles is much greater in $A$, and despite the large number of particles over vesicles in $A$, the background nonspecific labeling is much less. Little label is found over rough endoplasmic reticulum $(C)$ or Golgi apparatus $(G A)(E)$, but label is found over the large organelle in $D$ (arrow) with the appearance of a multivesiculated body. Colloidal gold label is generally found over the dense core of the neurosecretory vesicle but sometimes can be found slightly outside the vesicle; this may be due to the use of a secondary antibody adsorbed to gold. The two immunoglobulins between the antigen and the colloidal gold may displace the label approximately $15 \mathrm{~nm}$ from the antigen. Bars: $A$ and $B, 325 \mathrm{~nm} ; C, 250 \mathrm{~nm}, D, 325 \mathrm{~nm}$; $E, 350 \mathrm{~nm}$. 

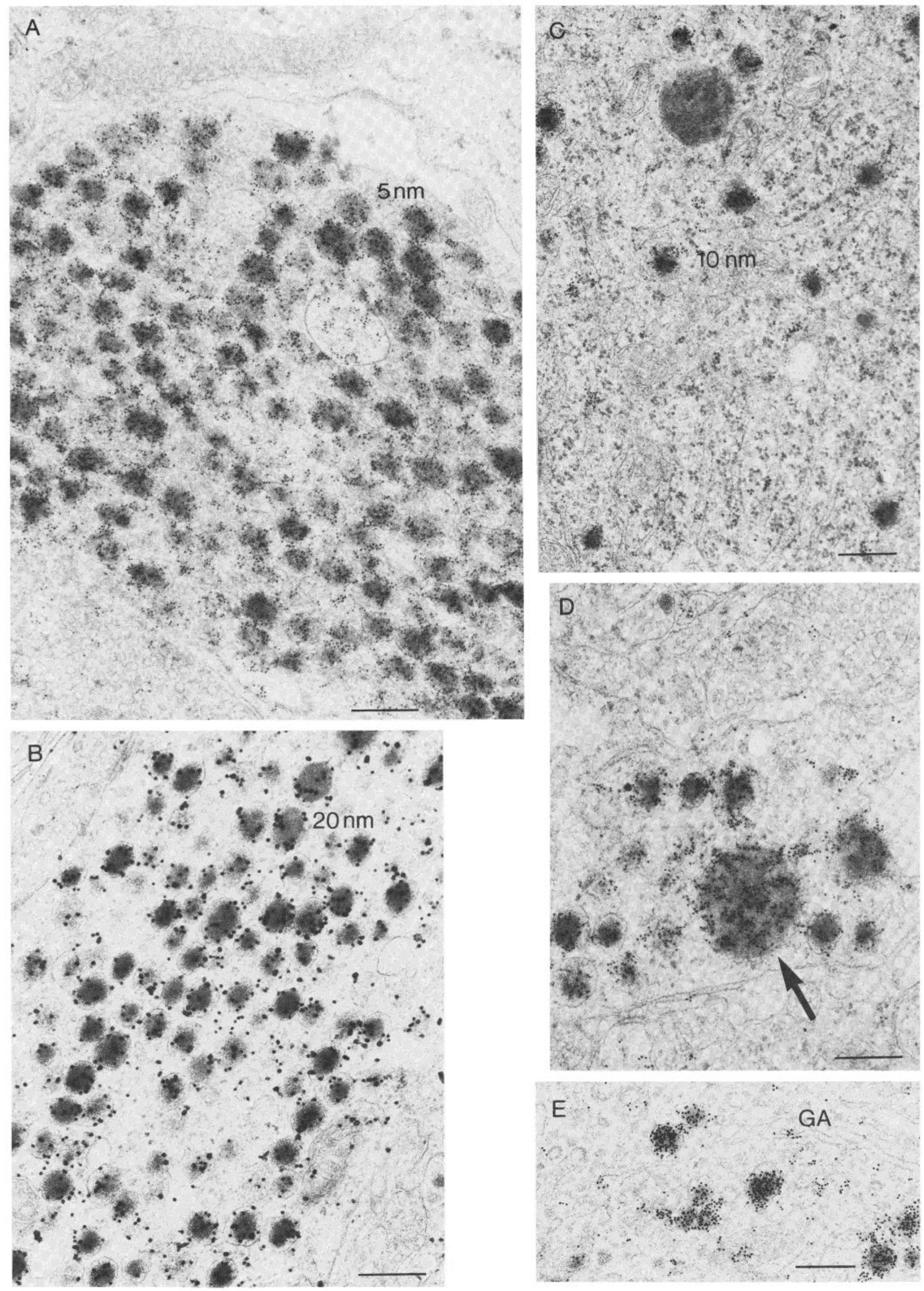
immunoreactive profiles were found in the preoptic area, consistent with previous reports (Rethelyi et al., 1981; King et al., 1982), no immunoreactive profiles were found within the SON (Fig. 1C).

Post-embedding immunostaining. With post-embedding immunostaining, ultrastructure, particularly at low electron microscopic magnifications, was more difficult to interpret with tissue embedded in Lowicryl K4M compared with other plastics; this was predominantly due to the negative membrane staining generally found with the embedding procedure used, whether or not the tissue was osmi- cated, rather than poor preservation. In contrast, the number of immunogold particles in non-osmicated tissue was greater in Lowicryl K4M (Fig. 2, C and D) than in Epon. Comparable staining levels over neurohypophyseal neurosecretory vesicles were found with Lowicryl K4M at a primary antisera dilution of $1: 1600$ as compared with 1:800 for Epon. LR White allowed strong immunostaining (Fig. $2, A$ and $B$ ), but contrast and ultrastructure seemed slightly better in Epon.

Of the four plastics used, tissue embedded in Epon or Durcupan

Figure 5. GAD-immunoreactive terminals synapsing on unlabeled dendrites. In $A, B$, and $C$ peroxidase/GAD-immunoreactive boutons make symmetrical synaptic contact (black arrows) with unlabeled dendrites. Unlabeled boutons also make synaptic contact with unlabeled dendrites. Small dense-core vesicles in A (hollow arrow) have no gold label. Background gold label is no greater over peroxidase-stained GAD boutons than over the nonstained adjacent neuropil. Bar, $250 \mathrm{~nm}$.

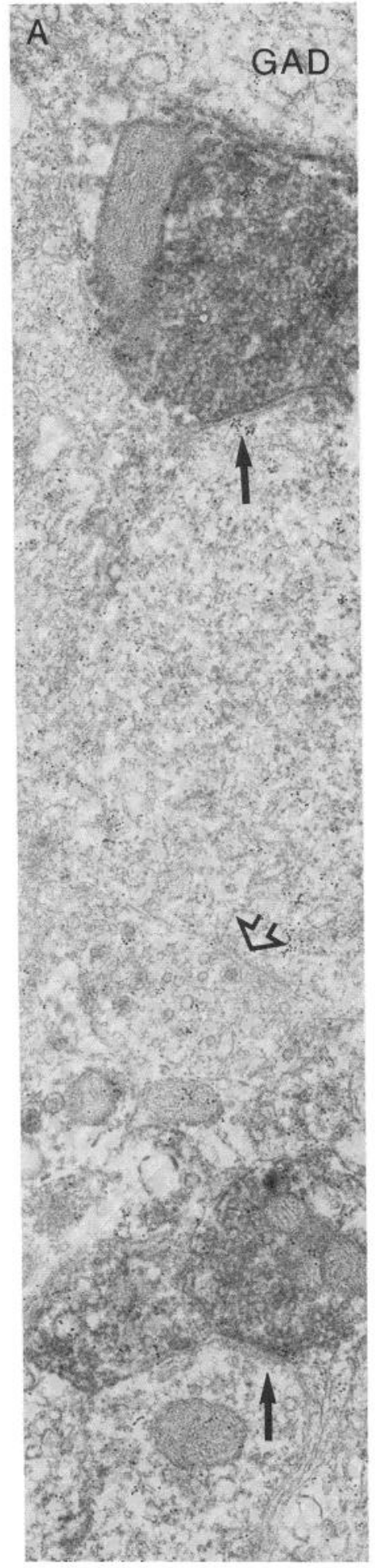

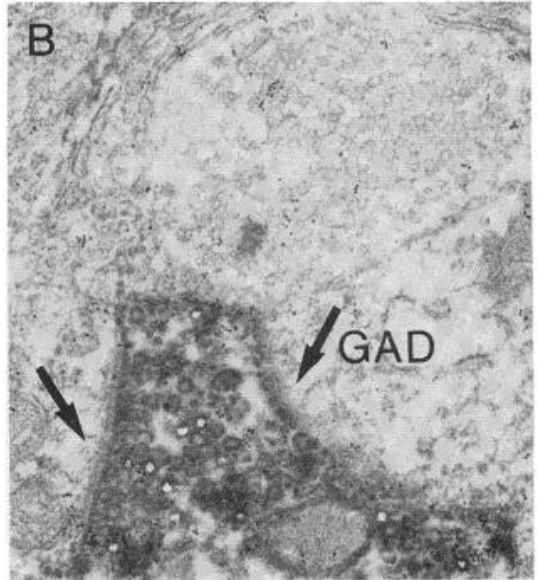

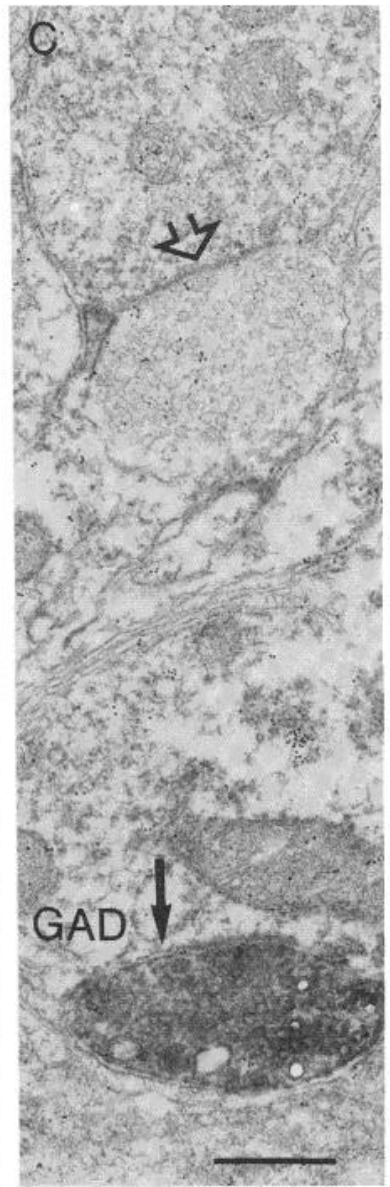


offered the best visualization of organelles, membranes, and synapses after post-embedding immunostaining. Because the primary objective of the present work was to study synaptic interactions between elements immunolabeled with different markers, Epon was selected for double-labeling experiments. Neurosecretory vesicles from unosmicated material generally had more gold particles than did those from osmicated tissue; however, as the osmicated tissue was much easier to examine with the electron microscope and the peroxidase labeling was more apparent, osmicated tissue was generally used for double labeling. Treatment of osmicated tissue with hydrogen peroxide was effective in increasing the number of immunogold particles over neurosecretory vesicles, but also resulted
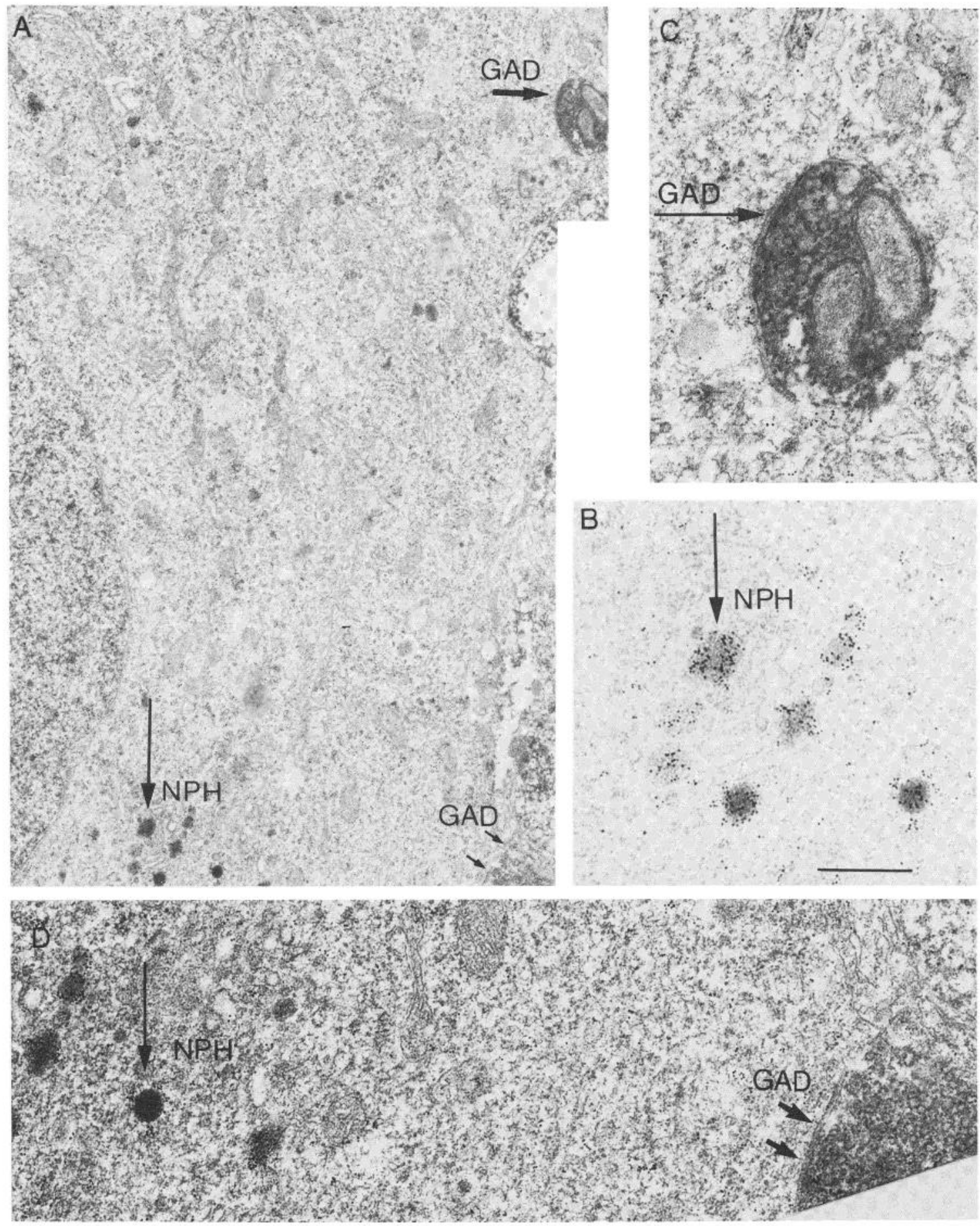

Figure 6. Double immunostaining. A, Two GAD-immunoreactive peroxidase-labeled terminals (horizontal and double arrows) terminate on a perikaryon in the SON. Ten nm gold particles are found over scattered NPH-immunoreactive neurosecretory vesicles (vertical arrow). The density of colloidal gold particles in this cell per unit area over neurosecretory vesicles containing NPH is 61 times greater than over the cytoplasm (after subtraction of Epon background labeling). $B$. Higher magnification ( $b a r=350 \mathrm{~nm}$ ) shows gold particles over vesicles. The vertical arrow indicates the same vesicle indicated by the vertical arrow in $A$. C, Higher magnification of axon stained with peroxidase with GAD antiserum making symmetrical contact with the gold-labeled cell body. $D$, Higher magnification of GAD-immunoreactive terminal in contact with cell at double arrows. 
in a significant deterioration of recognizable ultrastructure (Fig. 3). Treatment of osmicated tissue with sodium periodate (Bendayan and Zollinger, 1983) appeared to facilitate staining intensity to a lesser degree than did hydrogen peroxide, but with better resultant ultrastructural preservation.

Colloidal gold-immunoreactive NPH was found in large densecore vesicles in the SON (and paraventricular nucleus) (Figs. 2, 3, and 4) and neurohypophysis (Figs. 2, 3, 4, and 10). Gold label was also found over large (about $500 \mathrm{~nm}$ in diameter) organelles with an appearance like that of multivesiculated bodies (Fig. 4D). Specific label was absent over small dense-core vesicles, lysosomes, clear vesicles, mitochondria, or other subccllular organelles. Gold was not seen over the Golgi apparatus (Fig. 4E) but was found over large dense-core vesicles in the lateral regions of the Golgi apparatus. The ratio of gold particles per unit area over secretory vesicles compared to adjacent cytoplasm varied from more than 200:1 to $25: 1$, depending on fixation, type of plastic, length of incubation, etching, and reagent concentration (Figs. $2 B$ and $3 B$ ). Another important factor in labeling density was the size of the gold particle; smaller gold gave a high ratio of specific to nonspecific staining. More than 250 colloidal gold particles of a $5-\mathrm{nm}$ size could be found over single neurosecretory vesicles (Figs. $2 B$ and $3 C$ ), compared with less than 10 particles of $20 \mathrm{~nm}$ size (Fig. $4 B$ ).

Double immunolabeling. GAD-immunoreactive axons made numerous synaptic contacts with dendrites (Fig. 5) and with neuronal perikarya. Typical of GABA terminals elsewhere, the synaptic region had the general appearance of symmetrical synapses; small clear vesicles often filled the presynaptic bouton. Peroxidase reaction product was distributed throughout the bouton. An occasional dense-core vesicle was found among the smaller clear ones. Other boutons that were not immunoreactive for GAD antiserum also made synaptic contact; these boutons often had small dense-core vesicles and sometimes made asymmetrical synapses (Fig. 5, $A$ and $C$ ).

GAD-immunoreactive axons identified with peroxidase (Fig. 6) were found in synaptic contact with cell bodies and proximal dendrites of large cells containing large neurosecretory vesicles which were strongly NPH immunoreactive with colloidal gold particles. Synapses were of the symmetrical type with the same appearance as those immunoreactive boutons making synaptic contact with nonlabeled postsynaptic structures. GAD-immunoreactive terminals were commonly found contacting NPH-immunoreactive cells; cells were found which were contacted by two or three GAD-immunoreactive boutons in the same thin section.

To compare the labeling density of the colloidal gold particles over different structures after double labeling, a quantitative approach was used. At a magnification of $\times 78,750$, the number of gold particles over various structures was counted, and the area of the structure was measured with a Summagraphics Bit Pad Digitizer and a Bellco Projector. Fifteen samples were used for each different structure. The number of particles per unit area was compared (Fig. 7) based on the number of particles per $10,000 \mathrm{~nm}^{2}$. After subtracting the background Epon labeling $(0.19 \pm 0.18$ (SD) particles $/ 10,000$ $\mathrm{nm}^{2}$ ) from the mean $(0.42)$ of structures including mitochondria, lysosomes, nuclei, and cytoplasm, a tissue labeling background of 0.22 particles was obtained. This compares with the labeling of dense-core vesicles of $9.4 \pm 3.3$ particles; after subtraction of the Epon background, the dense-core vesicles had a labeling density of 9.21. Based on these numbers, the gold label over dense-core vesicles was 42 times greater than that over other tissue. The labeling of gold over peroxidase-stained GAD-immunoreactive boutons $(0.30$ \pm 0.25 (SD)) was no higher than any other non-immunoreactive structure, indicating a lack of cross-reactivity between the two series of immunoreagents.

Alternate thin section immunostaining for GABA and NPH. In order to maximize the number of structures stained with the preembedding peroxidase procedure, sections were frozen and thawed, and were left in various buffers for several days, resulting in some deterioration of ultrastructural preservation. In order to verify plasmalemmal continuity, and to confirm the synaptic nature of

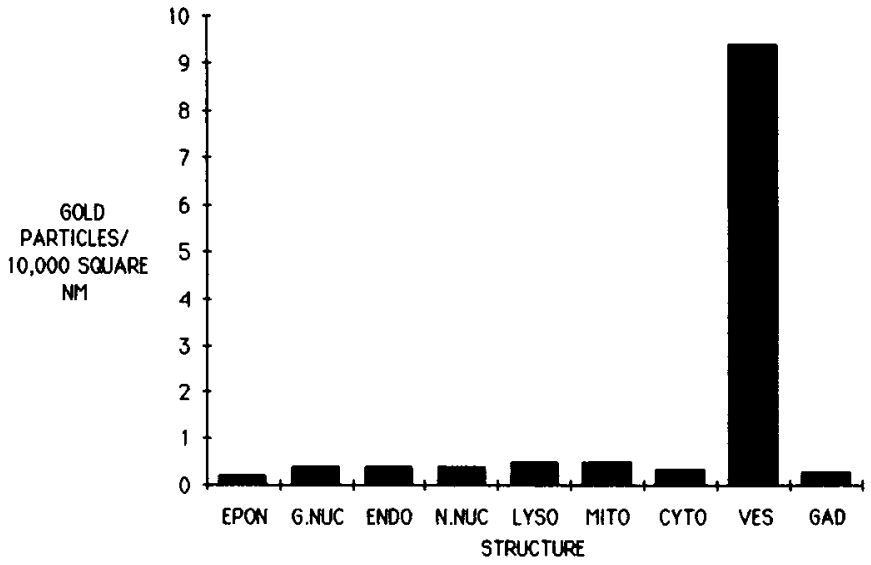

Figure 7. Colloidal gold density over different structures. Colloidal gold particles were counted over various structures from the grid used for double immunostaining in Figure 6. To standardize particle counts over structures of different sizes, the number of colloidal gold particles is expressed as number per $10,000 \mathrm{~nm}^{2}$. Epon-gold particles over Epon in the lumen of blood vessels. G.NUC, glial nucleus; ENDO, endothelial cell; N.NUC, neuron nucleus; LYSO, lysosome; MITO, mitochondrion; CYTO, cytoplasm; VES, large dense-core vesicle; $G A D$, peroxidase-labeled, GAD-immunoreactive bouton. Counts involving neuronal nuclei, lysosomes, mitochondria, and cytoplasm were all taken from neurons with immunoreactive dense-core vesicles. The standard deviations and the mean for each of the structures were: Epon, $0.19 \pm 0.18$; G.NUC, $0.42 \pm 0.10 ;$ ENDO, $0.38 \pm 0.11$; N.NUC, $0.37 \pm 0.12$; LYSO, $0.49 \pm 0.14 ;$ MITO, $0.50 \pm 0.23 ;$ CYTO, $0.34 \pm 0.16 ;$ VES, $9.4 \pm 3.3$; $\mathrm{GAD}, 0.30 \pm 0.25$.

contacts between GABAergic boutons and NPH-containing cells, serial thin sections were stained either with GABA or NPH antiserum. Post-embedding immunostaining may result in extraction of osmium and concommitant loss of membrane staining. To examine the synaptic nature of contacts between GABA- and NPH-immunoreactive elements, ultrathin serial sections were used with some sections stained only with the heavy metals uranyl acetate and lead citrate, or stained with GABA or with NPH antisera. Axonal boutons stained with colloidal gold and GABA antiserum were found making synaptic contact with cell bodies and dendrites within the SON (Fig. 8). Boutons stained with GABA antiserum and labeled with gold had the same general morphology as those stained in the SON with preembedding peroxidase and GAD antiserum. Gold particles were found over boutons making symmetrical synaptic contact with postsynaptic elements; gold particles were found over regions containing cytoplasm, small clear vesicles and over mitochondria, similar to the subcellular localization of GABA immunoreactivity with colloidal gold in cortical boutons (Somogyi and Hodgson, 1985). Neither postsynaptic dendrites nor somata were labeled; similarly, gold label was generally absent from boutons making asymmetrical synaptic contact. Not all boutons making symmetrical contact were labeled with GABA antiserum.

GABA-immunoreactive boutons were found in synaptic contact with perikarya (Fig. 9C) and proximal dendrites which, on adjacent serial sections, were shown to contain NPH immunoreactivity (9B). The synaptic contact zone was further verified in additional serial sections which had been stained only with lead and uranium (Fig. 9A).

Immunocytochemical controls. Deletion of the respective primary antisera, substitution of different sera (Fig. 1C), or the use of preimmune serum resulted in the absence of immunostaining with both the pre-embedding peroxidase GAD and GABA immunostaining and, similarly, with the post-embedding NPH immunogold staining. In the pituitary, immunogold staining with NPH antisera stained only the large dense-core granules in the neurohypophysis, site of the terminal projection of neurons of the SON (Fig. 10A); colloidal gold was not found over clear vesicles in the same axon containing large dense-core vesicles. Gold was also not found over other axon terminals lacking large dense-core vesicles, nor over boutons con- 

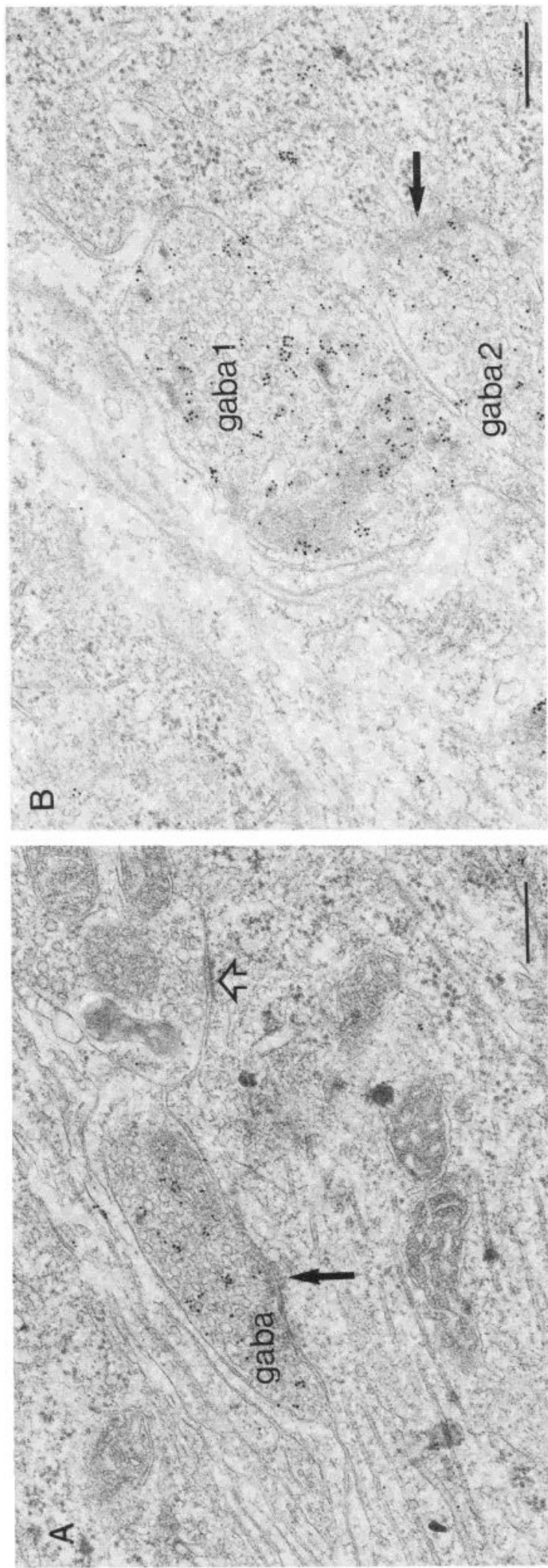

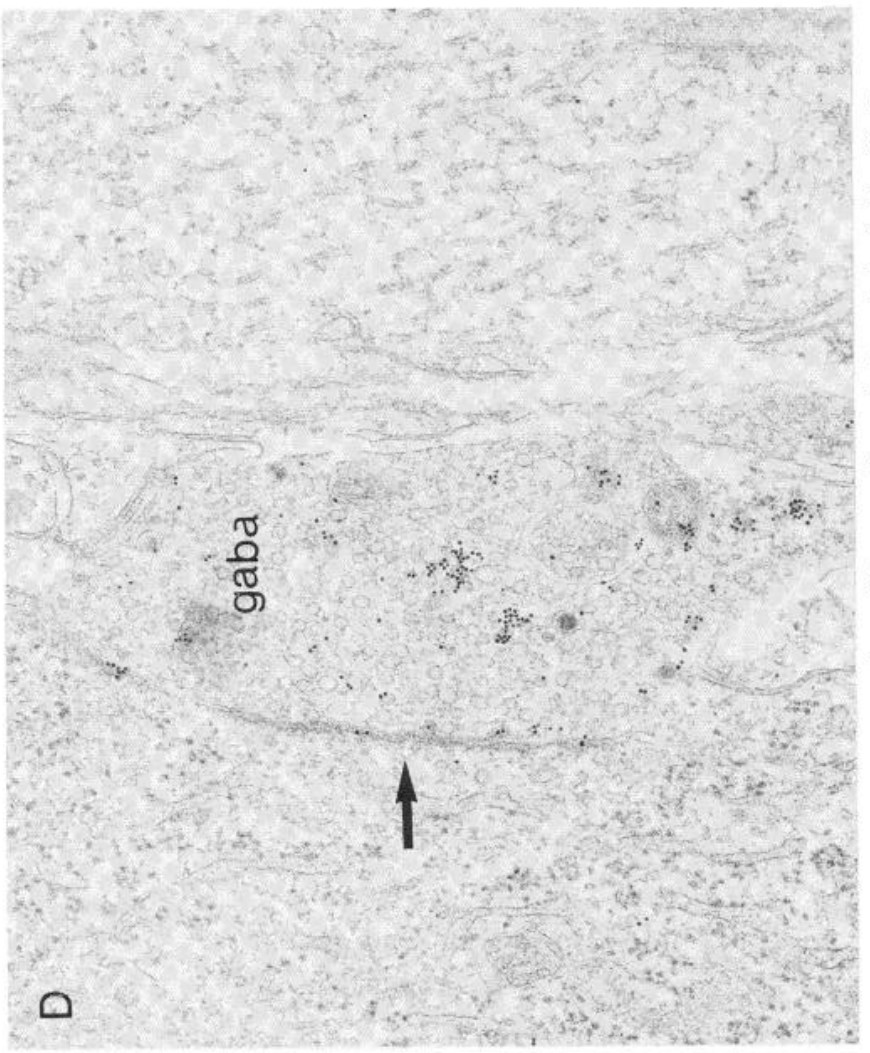

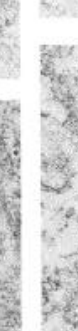
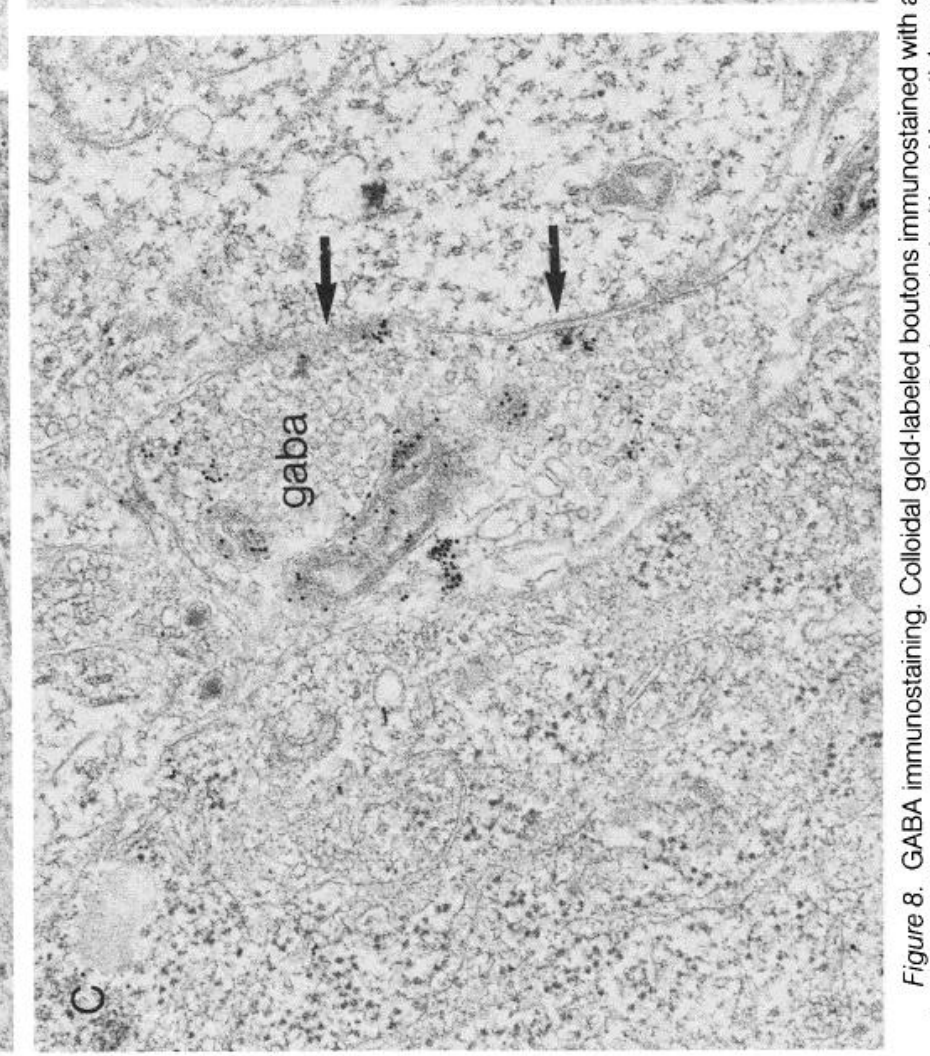

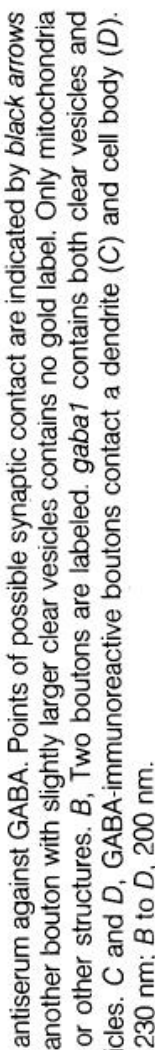



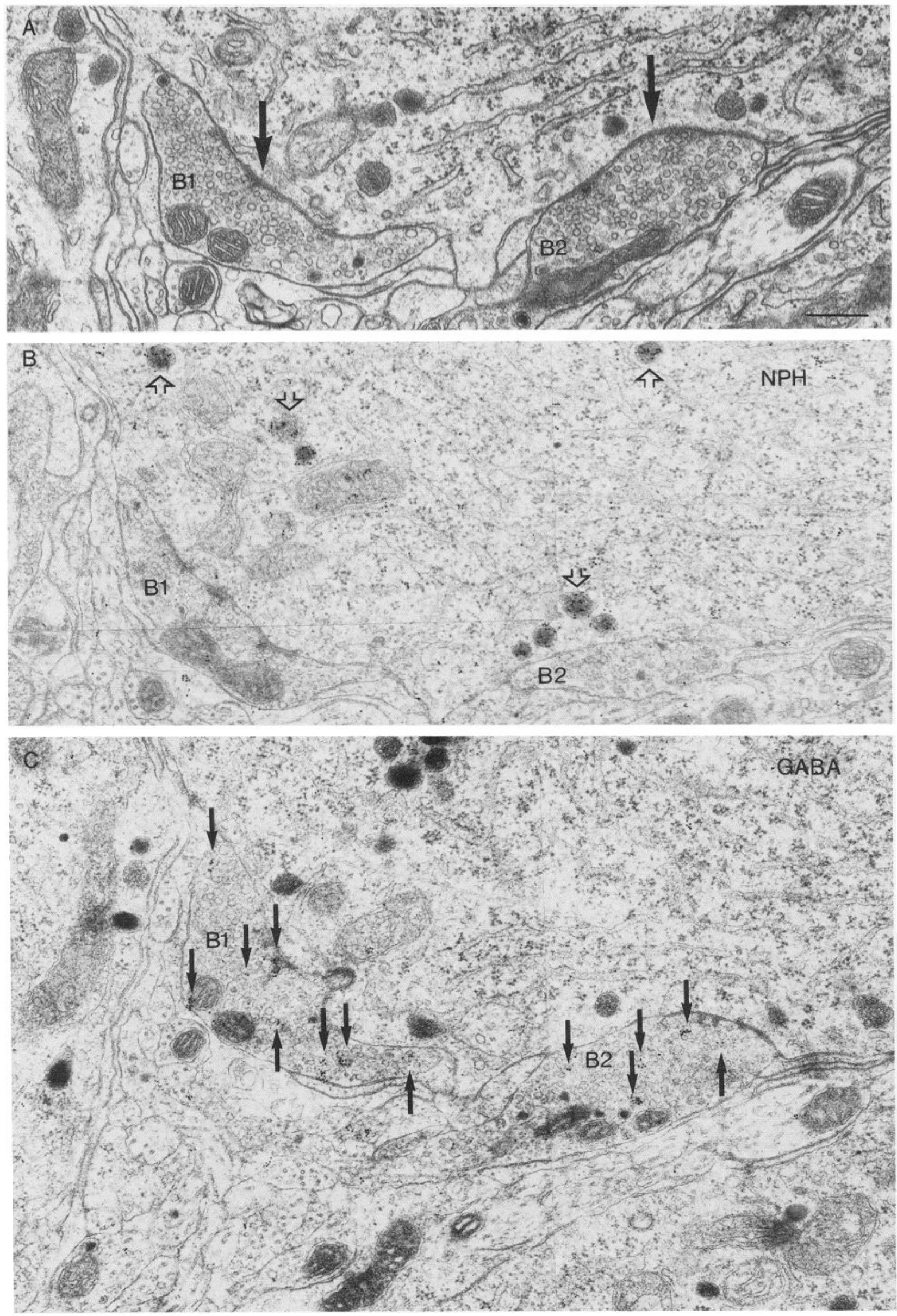

Figure 9. GABA bouton makes synaptic contact with NPH cell-serial sections. From a series of 80 serial ultrathin sections, alternate sections were used for immunostaining for GABA, NPH, or membrane staining. These three micrographs focus on a single pair of boutons (B1 and B2). $A$, Two boutons make symmetrical synaptic contact with a cell body in the SON. Arrows indicate regions of contact. B, postsynaptic cell contains large dense-core vesicles which 
taining small dense-core vesicles. Dense-core granules of the intermediate lobe and of the adenohypophysis were not immunolabeled (Fig. 10C), suggesting a specific staining of NPH rather than some other determinant associated with dense-core vesicles in general. In contrast, vesicles in the intermediate lobe were labeled when primary antisera against $\alpha$-melanocyte-stimulating hormone $(\alpha-\mathrm{MSH})$ or $\mathrm{ACTH}$ was used. Adsorption of the primary antisera with NPH antigen (UCB Bioproducts) blocked specific staining. To test specificity further, the primary antibodies were reversed; NPH immunostaining prior to embedding labeled large supraoptic neurons (Fig. 1A) but did not stain axons terminating on these cells. GAD antibody used after embedding, as expected, did not stain the large dense-core granules (Fig. 10D). With the Epon procedure used here, GADimmunoreactive axons were not labeled with post-embedding immunostaining procedures. Substitution of other plastics for Epon, including Lowicryl K4M, LR White, and Durcupan, gave similar staining specificity with post-embedding immunogold staining for $\mathrm{NPH}$.

GABA antisera used with post-embedding gold staining labeled the same type of bouton stained with peroxidase and pre-embedding immunostaining with GABA and GAD antisera. To prevent crossreaction of the GABA antiserum and the BSA carrier protein to which the GABA hapten was conjugated for antisera production, GABA antiscrum was used in the presence of $0.1 \%$ BSA; addition of BSA lowered the general background staining but did not block specific immunostaining. Preincubation of GABA antiserum with GABA (2 $\mathrm{mg} / \mathrm{ml}$ ) overnight greatly reduced immunostaining. Incubation of GABA antiserum with GABA-BSA conjugate eliminated specific immunostaining

Post-embedding colloidal gold immunostaining of other antigens. Since the versatility of the double staining method described here is in part based on the ability to stain antigens after Epon embedding, a number of other neurotransmitter-related antigens in addition to $\mathrm{NPH}$ were localized in axons in the medial hypothalamus and median eminence with the post-embedding procedure described here; these included ACTH, $\alpha$-MSH, prolactin, somatostatin, $\beta$-endorphin, and $\mathrm{LHRH}$. Pre-embedding peroxidase immunostaining with all of these substances resulted in reaction product distributed throughout the cytoplasm of immunoreactive cells. In contrast, with post-embedding staining, immunoreactivity with all of these substances was found exclusively in dense-core vesicles; LHRH immunoreactivity in densecore vesicles of axons in the median eminence is shown in Figure $10 E$.

\section{Discussion}

The data presented here are based on a double ultrastructural immunostaining approach to neurotransmitter identification of both pre- and postsynaptic neurons, and were confirmed with differential immunostaining of alternate serial ultrathin sections. The Discussion is divided into two sections: the first focuses on the general use of the double labeling approach, and the second examines the functional implications of GABAergic synapses on NPH-containing supraoptic neurons.

Immunocytochemistry. GAD/peroxidase-immunoreactive boutons were not labeled with colloidal gold after NPH immunostaining (Figs. $6, C$ and $D$, and 7 ), indicating that colloidal gold-adsorbed immunoglobulin or protein $A$ as a post-embedding immunocytochemical marker does not bind to the series of immunoreagents used with pre-embedding immunocytochemistry. This is true regardless of the species in which the antibodies were raised; neither homologous nor heterologous series of antisera cross-react, as demonstrated both in previous work with a rabbit antiserum to tyrosine hydroxylase in the hypothalamic paraventricular nucleus (van den Pol, 1983, 1984a) and in the present experiment with a sheep antiserum to GAD. This lack of cross-reaction is a distinct advantage in cases of double-labeling experiments and may be due to an insufficient exposure through Epon of immunoglobulins used in the pre-embedding steps to those used in the post-embedding steps, or to an excess of precipitated diaminobenzidine around the antibody complexes used in the pre-embedding steps.

The double-labeling approach in the present paper can be used best with antigens which can be readily localized with post-embedding immunostaining. To maximize both ultrastructural appearance and immunostaining, in the present set of double-labeling experiments, Epon has proven the most useful of the four plastics studied. In addition to NPH (van den Pol, 1984a) and GABA (Somogyi and Hodyson, 1985), a number of other hypothalamic putative neuroactive substances have been localized with the post-embedding colloidal gold procedure used here; these include $\mathrm{LHRH}$, prolactin, $\mathrm{ACTH}, \alpha-\mathrm{MSH}$, somatostatin, and $\beta$-endorphin; additionally, substance $P$, vasoactive intestinal polypeptide (Probert et al., 1981), and Met- and Leu-enkephalin (Larsson and Stengaard-Pedersen, 1982) have been found in the peripheral nervous system with postembedding colloidal gold procedures. Post-embedding immunostaining with antisera against tyrosine hydroxylase or GAD has worked poorly; other plastics, particularly Lowicryl K4M and probably AR White, may work better for localization of these cytoplasmic antigens. Alternatively, different methods of etching plastic may prove fruitful. The choice of plastics and fixatives is a complex one, and no single combination is universally better than another for all questions that can be addressed with an immunocytochemical approach.

Different size colloidal gold particles can be used for doublelabeling experiments with post-embedding immunocytochemistry (Geuze et al., 1981; Roth, 1982). This approach works best in homogenous tissue which can readily be prepared for histochemical analysis. The peroxidase pre-embedding/gold post-embedding procedure as described in the present paper offers an advantage in complex tissue like the central nervous system, in that an area of interest can be dissected out and studied with light microscopy after staining the first antigen with peroxidase. The visibility of the first antigen stained with peroxidase facilitates selection of the precisc area of interest for further ultrastructural study. Optimal fixation is compromised by several factors including the freeze-thawing before pre-embedding staining, the low glutaraldehyde level necessary for pre-embedding staining, and osmium extraction during the postembedding immunostaining steps. For ultrastructural surveys of large areas of tissue, peroxidase-labeled boutons can be found more easily and at lower magnifications than can gold-labeled boutons. However, because serial section double gold labeling does not require freeze-thawing and can tolerate higher levels of glutaraldehyde, better membrane preservation necessary for synapse definition may be obtained with the serial section double post-embedding approach. Unlike the pre-embedding/post-embedding procedure, the double post-embedding procedure requires that both antigens be immunoreactive after plastic embedding and osmication. As with pre-embedding staining with colloidal gold (van den Pol, 1985a, b), one can maximize the number of gold particles with post-embedding staining by reducing their size. Thus $3-n m$ particles would give the highest labeling density. However, in situations which require lower microscope magnifications for surveys of large areas of tissue, 3 $\mathrm{nm}$ particles are difficult to see. Another factor which may render small gold particies difficult to visualize is a section highly contrasted with lead citrate and uranyl acetate. Colloidal gold is most easily identified in sections will low inherenll contrast.

For immunolabeling of two cytoplasmic antigens which are less amenable to post-embedding immunostaining, we have used a

are immunoreactive for NPH. Boutons are not labeled with NPH antiserum. $C$, Both boutons ( $B 1$ and $B 2$ ) are labeled with gold particles with immunostaining with GABA antiserum. Some of the gold particles are indicated with small black arrows. Dense-core vesicles in the postsynaptic cell are not labeled when GABA is used as the primary antiserum. The density of gold labeling over the two boutons is more than 25 times greater than over other structures. Note the loss of membrane definition in $B$ and $C$ compared with the non-immunostained section in $A$. Bar: $225 \mathrm{~nm}$. 
Figure 10. Post-embedding immunogold staining controls. $A$, In the posterior pituitary, large dense-core vesicles containing NPH are labeled with gold particles. Mitochondria, clear vesicles, and cytoplasm are not labeled with $\mathrm{NPH}$ antiserum. Bar, $260 \mathrm{~nm}$ for $A, C$, and $D$. $B$, High magnification $(2.1 \times A)$ of two neurosecretory vesicles in the soma of a neuron in the SON. Alteration of the immunocytochemical staining parameters can increase particle density, but at the loss of easily visualized membranes, as seen here with many gold particles covering two vesicles. $C$, Control staining with $\mathrm{NPH}$ antiserum of the cells of the intermediate lobe of the pituitary which do not contain NPH. No specific gold label is seen. $D$, As a further check on immunogold staining specificity, GAD antiserum was substituted for NPH antiserum: no gold label is seen over neurosecretory vesicles in neurons of the SON. E, LHRH antiserum does not stain neurohypophysis, but does stain small vesicles in some axons (solid arrows) of the median eminence, but not others (hollow arrow).
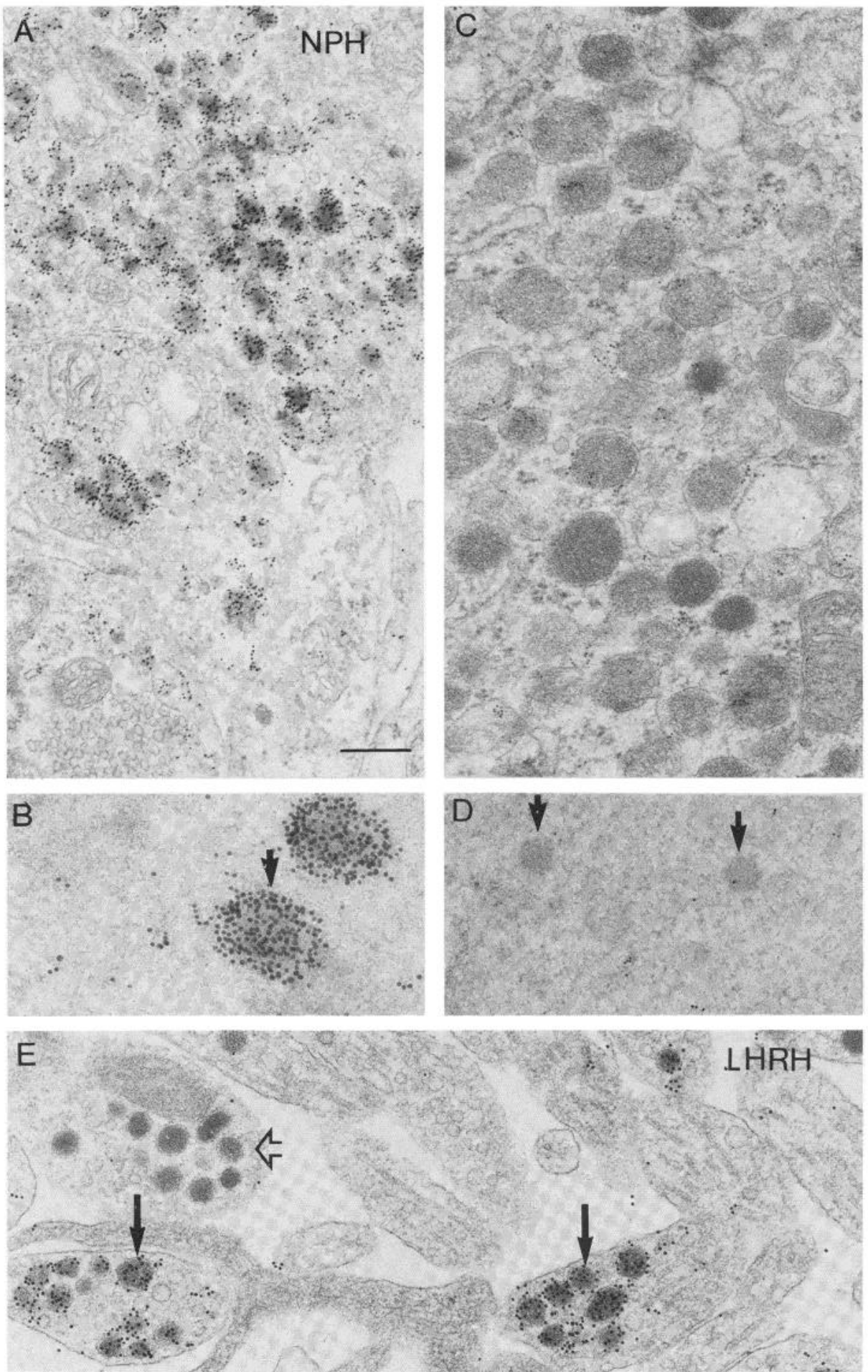

different approach from the one described here; silver-intensified gold (SIG), a black punctate label, can be combined with peroxidase, a diffuse brown label, for simultaneous visualization with both light and electron microscopy (van den Pol, 1984b, 1985a, b). Both the SIG/peroxidase procedure and the pre-/post-embedding procedures can be used with two primary antigens produced in the same animal, with little cross-reaction between the two series of immunoreagents, reducing the necessity of using primary antisera produced in different species for double labeling. Different factors contribute to the lack of cross-reaction in the two paradigms: with the double pre-embedding SIG/peroxidase protocol, the silver shell formed around the colloidal gold particle used with the first series of immunoreagents reduces the cross-reaction with a second series of reagents. With the pre-embedding peroxidase followed by the post-embedding gold procedure, the Epon effectively eliminates cross-reaction between two series of immunoglobulins or protein A.

Not only is a higher amount of glutaraldehyde tolerated in postembedding staining, but membrane damage mandatory for penetration of the large immunoglobulin molecules in pre-embedding staining is not necessary in post-embedding immunostaining. With postembedding staining, colloidal gold has an advantage over peroxidase in that, unlike peroxidase labeling, gold cannot be confused 
with the electron-dense matrix normally found in dense-core vesicles. Although only neurosecretory vesicles are specifically labeled. with post-embedding immunogold staining, with pre-embedding immunostaining for $\mathrm{NPH}$, $\mathrm{LHRH}$, somatostatin, $\mathrm{ACTH}, \alpha-\mathrm{MSH}$, and a number of other transmitter-related peptides, reaction product is often found throughout the cytoplasm (e.g., van den Pol, 1982, 1984a). This cytoplasmic labeling may be due to diffusion of the contents of vesicular membrane ruptured during or after weak fixation, or to membrane damage caused by detergents or freeze-thawing and subsequent staining of the translocated antigen. With a pre-embedding procedure, a concentration of $1 \%$ glutaraldehyde effectively blocks positive immunostaining. However, with post-embedding staining, the same concentration of glutaraldehyde works well. We have found sirnilar results when comparing pre- and post-ernbedding immunostaining of other substances, including $\mathrm{NPH}, \mathrm{LHRH}, \mathrm{ACTH}$, $\alpha-\mathrm{MSH}$, and prolactin. This suggests that the glutaraldehyde is not rendering determinants on these antigens unrecognizable by their homologous antisera, but rather is impeding diffusion of antisera into thick tissue sections by cross-linking cellular proteins.

Functional implications. Neurons of the SON are the most studied of neurons involved in endocrine control, and the best characterized of all hypothalamic neurons. Neurons here are the final common neuron involved in control and release of hormones into the vascular system. By working backwards from the final common immunocytochemically identified neuronal effector in this system, the nature of chemically defined control systems involved in neuroendocrine regulation can be elucidated, as was attempted in the present paper.

The presence of axons containing a number of peptide-neuroactive substances and catecholamines in the SON has been reviewed (Sawchenko and Swanson, 1982; Swanson and Sawchenko, 1983). GABA has for the most part received little attention. In the present study, not only is evidence for GABA found throughout the SON, but GABA also appears to be present in a large number of boutons terminating on NPH containing SON cells. Both pro cmbedding per oxidase immunostaining with GAD antiserum and post-embedding colloidal gold immunostaining with GABA antiserum revealed a substantial GABAergic innervation of neurons in the SON. To a large degree, the previous lack of morphological work on GABA here may have been due to the difficulty in purification of the GAD antigen (Oertel et al., 1981a, b; Wu, 1983) and to our past ignorance about making antisera against single amino acids such as GABA (StormMathisen et al., 1983; Hodgson et al., 1985). In contrast, catecholamine-containing neurons could be recognized 20 years ago by the classical fluorescence techniques (Falck et al., 1962).

In physiological studies microiontophoresis of GABA onto neurosecretory neurons of the SON and paraventricular nucleus consistently inhibited firing rates (Nicoll and Barker, 1971; Moss et al., 1972). Neurons of the SON, antidromically identified as projecting to the neurohypophysis, with electrophysiological characteristics of both oxytocin- and vasopressin-releasing cells, were inhibited by GABA iontophoresis (Bioulac et al., 1978). Similarly, in vitro superfusion of GABA had an inhibitory influence on neurons and axons of the SON (Sakai et al., 1974; Zingg et al., 1979; Mathison and Dreifuss, 1980). Release of oxytocin (Dyball and Shaw, 1979) and vasopressin (Feldberg and Rocha E Silva, 1978) was inhibited by GABA and increased by GABA antagonists, respectively. In the present study GAD- and GABA-immunoreactive boutons are found in all regions of the SON. GAD-immunoreactive presynaptic boutons not only are found in large numbers contacting cell bodies of neurosecretory neurons, but they also may constitute a major type of synapse on the cell body, as indicated in Results. GAD-immunoreactive axons are also found in large numbers making synaptic contact with unlabeled dendrites, many of which probably arise from magnocellular neurons. The presence of large numbers of GABAand GAD-immunoreactive axons in synaptic contact with immunocytochemically identified neurosecretory neurons of the hypothalamic SON suggests that GABA may inhibit neural activity and release of the neurohormones oxytocin and vasopressin from the axon terminals in the neurohypophysis. The presence of GABA neurons in the immediate vicinity of the SON (Meyer et al., 1980; Tappaz et al., 1982) suggests that some of the GABA innervation of the SON may arise from local collaterals of nearby neurons. Since these cells would be near all of the major afferent inputs to the SON, local GABA-containing cells would be good candidates for modulating and coordinating firing of SON neurons. Similarly, parvocellular neurons of the medial paraventricular nucleus have local axons which terminate on the nearby lateral magnocellular neurons (van den Pol, 1982) and may influence oxytocin and vasopressin release from axons of that nucleus. The additional presence of GADimmunoreactive axons in the neurohypophysis (Oertel et al., 1982; Vincent et al., 1982) coupled with findings that axons in the neurohypophysis respond directly to GABA (Zingg et al., 1979; Mathison and Dreifuss, 1980) provides further support for a role of GABA in the modulation of release of the neurohormones oxytocin and vasopressin

\section{References}

Altman, L., B. G. Schneider, and D. S. Papermaster (1984) Rapid embedding of tissues in Lowicryl K4M for immunoelectron microscopy. J. Histochem. Cytochem. 32: 1217-1223.

Bendayan, M., and M. Zollinger (1983) Ultrastructural localization of antigenic sites on osmium-fixed tissue applying the protein A-gold technique. J. Histochem. Cytochem. 31: 101-109.

Bioulac, B., B. Dufy, F. DeVitry, H. Fleury, A. Tixier-Vidal, and J. D. Vincent (1978) Effects of acetylcholine, sodium glutamate, and GABA on the discharge of supraoptic neurons in the rat. Brain Res. 154: 159-162.

Buijs, R. (1978) Intra- and extra-hypothalamic vasopressin and oxytocin pathways in the rat. Pathways to the limbic system, medulla oblongata and spinal cord. Cell Tissue Res. 192: 423-435.

DeMey, J., M. Moeremans, G. Geuens, R. Nuydens, and M. DeBrabander (1981) High resolution light and electron microscopic localization of tubulin with the IGS (immuno gold staining) method. Cell Biol. Int. Rep. 5: 889 899

Dyball, R. E. J., and F. D. Shaw (1979) Inhibition by GABA of hormone release from the neurohypophysis in the rat. J. Physiol. (Lond.) 289: 78P. $79 \mathrm{P}$

ralck, B., N. A. l lillarp, G. Thieme, and A. Torp (1962) rluorescence of catecholamines and related compounds condensed with formaldehyde. J. Histochem. Cytochem. 10: 348-354

Feldberg, W., and M. Rocha E. Silva (1978) Vasopressin release produced in anaesthetized cats by antagonists of gamma-aminobutyric acid. Br. J. Pharmacol. 62: 99-106.

Felten, D. L., and K. A. Cashner (1979) Cytoarchitecture of the supraoptic nucleus: A Golgi analysis. Neuroendocrinology 29: 221-230.

Frens, G. (1973) Controlled nucleation for the regulation of the particle size in monodisperse gold solutions. Nature Phys. Sci. 241: 20-22.

Geuze, J., J. W. Slot, P. A. van der Ley, and R. C. T. Scheffer (1981) Use of colloidal gold particles in double-labelling immunoelectron microscopy of ultrathin frozen tissue sections. J. Cell Biol. 89: 653-665.

Hodgson A. J., B. Penke, A. Erdej, I. W. Chubb, and P. Somogyi (1985) Antisera to gamma amino butyric acid. I. Froduction and characterization using a new model system. J. Histochem. Cytochem. 33: 229-239.

Holgate, C. S., P. Jackson, P. N. Cowen, and C. C. Bird (1983) Immuno-gold silver staining: A new method of immunostaining with enhanced sensitivity. J. Histochem. Cytochem. 31: 938-944.

Horisberger, M. (1979) Evaluation of colloidal gold as a cytochemical marker for transmission and scanning electron microscopy. Biol. Cell. 36: 253258

Horisberger, M., and M. Vonlanthen (1978) Simultaneous localization of a hepatic binding protein specific for galactose and of galactose-containing receptors on rat hepatocytes. J. Histochem. Cytochem. 26: 960-966.

Hsu, S., L. Raine, and H. Fanger (1981) Use of avidin-biotin-peroxidase complex $(A B C)$ in immunoperoxidase techniques: A comparison between $\mathrm{ABC}$ and unlabeled antibody (PAP) procedures. J. Histochem. Cytochem. 29: 577580

King, J. C., S. A. Tobet, F. L. Snavely, and A. A. Arimura (1982) LHRH immunopositive cells and their projections to the median eminence and urganum vasculosum of the lamina terminalis. J. Comp. Neurol. 209: 287300.

Larsson, L. I., and K. Stengaard-Pedersen (1982) Immunocytochemical and ultrastructural differentiation between Met-enkephalin-, Leu-enkephalin-, and Met/Leu-enkephalin-immunoreactive neurons of feline gut. J. Neurosci. 2: $861-878$ 
LuQui, I. J., and C. A. Fox (1976) The supranptic nucleus and the supraopticoneurohypophyseal tract in the monkey (Macaca mulatta). J. Comp. Neurol. 168: $7-40$.

Mathison, R. D., and J. J. Dreifuss (1980) Structure activity rclationships of a neurohypophyseal GABA receptor. Brain Res. 187: 476-480.

Meyer, D. K., W. H. Oertel, and M. J. Brownstein (1980) Deafferentation studies on the glutamic acid decarboxylase content of the supraoptic nucleus of the rat. Brain Res. 200: 165-168.

Moss, R. L., I. Urban, and B. A. Cross (1972) Microelectrophoresis of cholinergic and aminergic drugs on paraventricular neurons. Am. J. Physiol. 223: $310-318$.

Nicoll, R. A., and J. L. Barker (1971) The pharmacology of recurrent inhibition in the supraoptic neurosecretory system. Brain Res. 35: 501-511.

Nilaver, G., E. A. Zimmerman, J. Wilkins, J. Michaels, D. Hoffman, and A. J. Silverman (1980) Magnocellular hypothalamic projections to the lower brain stem and spinal cord of the rat. Neuroendocrinol. 30: 150-158.

Oertel, W. H., D. E. Schmechel, M. L. Tappaz, and I. Kopin (1981a) Production of a specific antiserum to rat brain glutamic acid decarboxylase by injection of an antigen-antibody complex. Neuroscience 6: 2689-2700.

Oertel, W.H., D. E. Schmechel, E. Mugnaini, M. L. Tappaz, and I. J. Kopin (1981b) Immunocytochemical localization of glutamate decarboxylase in rat cerebellum with a new antiserum. Neuroscience 6, 2715-2735.

Oertel, W. H., E. Mugnaini, M. L. Tappaz, V. K. Weise, A. Dahi, D. E. Schmechel, and I. J. Kopin (1982) Central GABAergic innervation of neurointermediate pituitary lobe: Biochemical and immunocytochemical study in the rat. Proc. Natl. Acad. Sci. U. S. A., 79: 675-679.

Probert, L., J. DeMey, and J. M. Polak (1981) Distinct subpopulations of enteric $p$-type neurones contain substance $P$ and vasoactive intestinal polypeptide. Nature 294: 470-471.

Rethelyi, M., S. Vigh, G. Setalo, I. Merchenthaler, B. Flerko, and P. Petrusz (1981) The luteinizing hormone releasing hormone-containing pathways and their co-termination with tanycyte processes in and around the median eminence and in the pituitary stalk of the rat. Acta Morphol. Acad. Sci. Hung. 29: 259-283.

Romano, E. L., C. Stolinsky, and C. Hugh-Jones (1974) An antiglobulin reagent labelled with colloidal gold for use in electron microscopy. Immunochemistry 11: 521-522.

Roth, J., M. Bendayan, and L. Orci (1978) Ultrastructural localization of intracellular antigens by the use of protein A gold complex. J. Histochem. Cytochem. 26: 1074-1081

Sakai, K. K., B. H. Marks, J. M. George, and A. Koestner (1974) The isolated organ-cultured supraoptic nucleus as a neuropharmacogogical test system. J. Pharmacol. Exp. Ther. 190: 482-491.

Sawchenko, P. E., and L. W. Swanson (1982) The organization of noradrenergic pathways from the brainstem to the paraventricular and supraoptic nuclei in the rat. Brain Res. Rev. 4: ?75-325

Sofroniew, M. V., and A. Weindl (1978) Extrahypothalamic neurophysin containing perikarya, fiber pathways, and fiber clusters in the rat brain. Endocrinology 102: 334-337

Somogyi, P., and Hodgson, A. J. (1985) Antiserum to $\gamma$-amino butyric acid. III. Demonstration of GABA in Golgi-impregnated neurons and in conventional electron microscopic sections of cal striale corlex. J. Histocherr.
Cytochem. 33: 249-257.

Sternberger, L. A. (1979) Immunocytochemistry. John Wiley \& Sons, Inc., New York.

Storm-Mathisen, J., A. K. Leknes, A. T. Bore, J. L. Vaaland, P. Edminson, F. M. Haug, and O. P. Ottersen (1983) First visualization of glutamate and GABA in neurones by immunocytochemistry. Nature 301: 517-520.

Swanson, L. W., and P.E. Sawchenko (1983) Hypothalmic integration: Organization of the paraventricular and supraoptic nuclei. Ann. Rev. Neurosci. 6: 269-324.

Tappaz, M. L., W. H. Oertel, M. Wassef, and E. Mugnaini (1982) Central GABAergic neuroendocrine regulations: Pharmacological and morphological evidence. Prog. Brain Res. 55: 77-96.

Tappaz, M. L., M. Wassef, W. H. Oertel, L. Paut, and J. F. Pujol (1983) Lightand electron-microscopic immunocytochemistry of glutamic acid decarboxylase (GAD) in the basal hypothalamus: Morphological evidence for neuroendocrine gamma-aminobutyrate (GABA). Neuroscience 9: 271287.

Toubcau, G., J. Desclin, M. Parmentier, and J. L. Pasteels (1979) Cellular localization of a prolactin-like antigen in the rat brain. J. Endocrinol. 83: 261-266.

varı den Pol, A. N. (1982) The magnocellular and parvocellular paraventricular nucleus of rat: Intrinsic organization. J. Comp. Neurol. 206: 317-345.

van den Pol, A. N. (1983) Colloidal gold and peroxidase as ultrastructural markers for simultaneous localization of two neurotransmitter antigens in the hypothalamic paraventricular nucleus. Soc. Neurosci. Abstr. 9: 84.

van den Pol, A. N. (1984a) Colloidal gold and biotin-avidin conjugates as ultrastructural markers for neural antigens. Q. J. Exp. Physiol. 69: 1-33.

van den Pol, A. N. (1984b) Double pre-embedding ultrastructural immunocytochemistry with intensified colloidal gold and peroxidase: Dopamine neurons in the dorsomedial hypothalamus receive GABA synapses. Soc. Neurosci. Abstr. 10: 440 .

van den Pol, A. N. (1985a) Silver intensified gold and peroxidasc as dual immunolabels for simultaneous visualization of pre- and postsynaptic neurotransmitters. Science 228: 332-335.

van den Pol, A. N. (1985b) Tyrosine hydroxylase-immunoreaclive neurons in the hypothalamus receive glutamate decarboxylase-immunoreactive synapses: A double pre-embedding immunocytochemical study with particulate silver and horseradish peroxidase. J. Neurosci., in press.

van den Pol, A. N., and K. Tsujimoto (1985) Neurotransmitters of the hypothalamic suprachiasmatic nucleus-Immunocytochemical analysis of 25 neuronal antigens. Neuroscience 15: 1049-1086.

van den Pol, A. N., R. S. Herbst, and J. F. Powell (1984) Tyrosine hydroxylase immunoreactive neurons of the hypothalamus: A light and electron microscopic study. Neuroscience 13: 1117-1156.

Vincent, S. R., T. Hokfelt, and J. Y. Wu (1982) GABA neuron systems in hypothalamus and the pituitary gland. Neuroendocrinology 34: 117-125.

Wu, J. -Y. (1983) Preparation of glutamic acid decarboxylase as immunogen for immunocytochemistry. In Immunohistochemistry, C. Cuello, ed., pp. 159-191, John Wiley \& Sons, Inc., New York.

Zingg, H. H., A. J. Baertschi, and J. J. Dreifuss (1979) Action of gammaaminobutyric acid on hypothalamo-neurohypophyseal axons. Brain Res. 171: $453-459$ 